\title{
Elevated Pressure Aqueous Hemostasis: Experimental and Mathematical Modeling
}

Matt T Oberdier* and James F Antaki

Department of Biomedical Engineering, Carnegie Mellon University, USA

\begin{abstract}
Elevated Pressure Aqueous Hemostasis is defined as the use of hydrostatic pressure via an isotonic liquid medium in a closed surgical field to control bleeding. It is an effective yet under-characterized means of achieving hemostasis; however, compromised perfusion is a potential complication. Therefore, the objective of this study was to determine the optimal range of extravascular pressure that both limits hemorrhage and allows antegrade flow. A steady-state experimental flow system was employed to simulate series arterial and venous hemodynamics, venous collapse, and arterial hemorrhage. A corresponding lumped-parameter mathematical model, calibrated to experimental data, was then used to extrapolate to conditions of hypotension, normotension, hypertension, limited venous collapse, venous hemorrhage, and simultaneous arterial and venous hemorrhages. Experiments with an elastomeric phantom vessel showed that hemorrhage from a stab incision was diminished with increasing extravascular pressure but was accompanied by decreased antegrade flow due to venous collapse. Above arterial pressure, flow ceased. Hence, a preferred pressure domain for aqueous hemostasis was defined to be greater than venous pressure to reduce bleeding and at least ten $\mathrm{mmHg}$ below arterial pressure to allow antegrade flow. Results from the lumped-parameter model suggest that i) a tethered vein may permit more antegrade flow for a given extravascular pressure; and ii) an elevated extravascular pressure in the presence of a venous rent may cause intravasation. A set of indices of perfusion and hemorrhage were introduced to generalize these results and suggest guidelines for clinical practice.
\end{abstract}

Keywords: Mathematical modeling; Elevated pressure; Hemostasis; Lumped-parameter modeling

\section{Introduction}

Minimally invasive surgical techniques are increasingly becoming a preferred alternative to open procedures [1]. Many closed procedures utilize $\mathrm{CO}_{2}$ insufflation to distend the operative space [2]; however, isotonic solutions are also used. Examples are found in urology [3-6], gynecology $[7,8]$, orthopedics $[9,10]$, and neurosurgery [11-18] in which visualization is facilitated by resectoscope, hysteroscope, arthroscope, and ventriculoscope, respectively. An aqueous environment distends the operative field $[6,9]$, allows for hemostasis $[4,5,9,10]$, and improves visual clarity via irrigation $[3,6,9,11,15-18]$. An additional benefit of an aqueous versus gaseous medium is the eliminated risk of air embolism [3].

Titled here as Elevated Pressure Aqueous Hemostasis (EPAH), the purposeful introduction of a pressurized aqueous environment into a closed surgical field to control hemorrhage has been demonstrated to staunch the egress of blood from open vessels $[4,5,9,10]$. However, an inevitable effect of an aqueous procedure is the application of elevated hydrostatic pressure throughout the surgical field, which may cause vascular collapse. These secondary effects have not heretofore been studied, and consequently there are no standard guidelines for regulating the aqueous pressure.

Therefore, the purpose of the present study was to characterize the balance between the beneficial effect of limiting hemorrhage and the potentially harmful effect of inhibiting antegrade flow. An ultimate objective is to define an optimal range of elevated extravascular pressure that may provide efficacy without introducing unacceptable risk.

\section{Methods}

This study involved complementary experimental and theoretical components. Experiments were performed to calibrate and validate a lumped-parameter model. Both the fluid circuit and electrical analog correspond to a simplified series arteriovenous circulation under steady flow conditions. Table 1 is a guide of the variables used throughout this study.

\section{Experiments}

A study was conducted that utilized a simplified model of EPAH, comprised of an elastomeric mock artery and vein within a sealed, rigid enclosure (Figure 1). The diameter, thickness, and durometer of the tubes were chosen to match those of the abdominal aorta and inferior vena cava [19] with considerations for fluid dynamic scaling [20] and prior studies of collapsible tubes [21-23]. The arterial side featured a thick- walled latex rubber tube (1/2" ID and 5/8" OD; 5234K261; Bard Medical Division; Covington, GA) 5/8" in diameter and cut to the same length. The tubes were oriented horizontally and connected to $1 / 2$ " barbs (A-387; Watts Regulatory Company; North Andover, MA) secured by cable ties with silicone rubber jackets at each junction to prevent leakage. The tubing barbs transversed the lateral walls of the chamber by sealed bulkhead connectors. The chamber was constructed from $3 / 8$ " thick acrylic to minimize bowing and was sized in an 11 " $\times 11$ " $\times 4$ " $(\mathrm{L} \times \mathrm{W} \times \mathrm{H})$ configuration, and a removable upper cover (attached with 22 machine screws) allowed access for exchanging tubes. A rigid septal wall was introduced to provide further support with 2" x 1" (L x H) cutouts in each corner to allow pressure equilibrium.

The external fluid circuit consisted of three reservoirs suspended at different heights corresponding to arterial, venous, and extravascular pressures and connected to one end of the mock artery, vein, and

*Corresponding author: Matt T Oberdier, Department of Biomedical Engineering Carnegie Mellon University, Pittsburgh, PA 15213, USA, Tel: +412 268 9857; E-mail: mattoberdier@hotmail.com

Received September 17, 2013; Accepted November 04, 2013; Published November 11, 2013

Citation: Oberdier MT, Antaki JF (2013) Elevated Pressure Aqueous Hemostasis: Experimental and Mathematical Modeling. J Bioengineer \& Biomedical Sci 3: 126. doi: 10.4172/2155-9538.1000126

Copyright: @ 2013 Oberdier MT, et al. This is an open-access article distributed under the terms of the Creative Commons Attribution License, which permits unrestricted use, distribution, and reproduction in any medium, provided the original author and source are credited. 
extravascular pressure ports, respectively. The artery and vein were connected in series through a variable fluidic resistor (46425K14; McMaster-Carr; Robbinsville, NJ). A centrifugal pump (4E-34NR; Little Giant Pump Company; Oklahoma City, OK) continuously recirculated fluid from the venous to the arterial reservoir. A check valve (RetroGuard ; QUEST ${ }^{\oplus}$ Medical Incorporated; Allen, TX) was interposed between the reservoir and chamber on the arterial side to prevent retrograde flow. Ball valves (R661; Homeworks Worldwide; Wheeling, IL) were provided in each of the supply lines to isolate the chamber.

The extravascular fluid was standard tap water while the circulating fluid was a blood analog fluid consisting of an approximately 60:40 mixture of tap water and glycerin (Fisher Acros Organics; Somerville,

\begin{tabular}{|c|c|}
\hline Variable & Description \\
\hline$P_{A, i}$ & Arterial Inlet Pressure \\
\hline$P_{A, 0}$ & Arterial Outlet Pressure \\
\hline$P_{V, i}$ & Venous Inlet Pressure \\
\hline$P_{V, O}$ & Venous Outlet Pressure \\
\hline$P_{E V}$ & Extravascular Pressure \\
\hline$P_{T M, A}$ & Arterial Transmural Pressure \\
\hline$P_{T M, V}$ & Venous Transmural Pressure \\
\hline$P_{C}$ & Critical Closing Pressure \\
\hline$Q_{A}$ & Arterial Flow \\
\hline$Q_{A V}$ & ArterioVenous Flow \\
\hline$Q_{V}$ & Venous Flow \\
\hline$Q_{H}$ & Hemorrhage Flow \\
\hline$R_{i}$ & Inlet Resistance \\
\hline$R_{A}$ & Arterial Resistance \\
\hline$R_{A R}$ & Arterial Rent Resistance \\
\hline$R_{A V}$ & ArterioVenous Resistance \\
\hline$R_{V R}$ & Venous Rent Resistance \\
\hline$R_{V}$ & Venous Resistance \\
\hline$R_{o}$ & Outlet Resistance \\
\hline$H I$ & Hemorrhage Index \\
\hline$C l$ & Circulation Index \\
\hline$A H I$ & Aqueous Hemostasis Index \\
\hline
\end{tabular}

Table 1: Legend of variables and their corresponding descriptions. Not presented in the table are the subscripts 0 and $P$, which denote the baseline condition $\left(P_{\mathrm{EV}}=0 \mathrm{mmHg}\right)$ and the elevated extravascular pressure level, respectively.

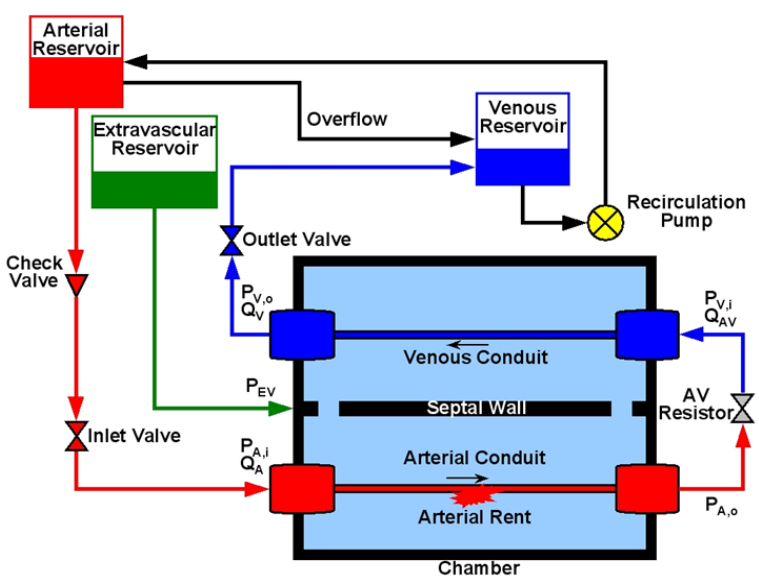

Figure 1: Schematic of the experiment including: arterial and venous conduits, chamber, arteriovenous resistor, reservoirs, recirculation pump, valves, three flow sensors $\left(Q_{A}, Q_{A V}\right.$, and $\left.Q_{V}\right)$, and five pressure tranducers $\left(P_{A, i}, P_{A, 0}, P_{V, i}, P_{V, 0}\right.$, and $\left.\mathrm{P}_{\mathrm{EV}}\right)$.
$\mathrm{NJ})$. The viscosity was titrated to $3.5 \pm 0.1 \mathrm{cP}$ and confirmed via capillary viscometer (C497; Cannon Instrument Company; State College, PA). To prevent microbial growth, a fungicide (Aquacide; Aquacide Waterbed and Spa Accessories; Auburn, CA) was introduced to the intravascular fluid at a concentration of approximately $1 \mathrm{~mL} / \mathrm{L}$.

Three turbine flow sensors (FT-110 173931-C; Gems Sensors and Controls; Palinville, CT) were located distal to the arterial tube $\left(\mathrm{Q}_{\mathrm{A}}\right)$, between the arterial and venous tubes $\left(\mathrm{Q}_{\mathrm{AV}}\right)$, and distal to the venous tube $\left(\mathrm{Q}_{\mathrm{V}}\right)$ and connected to respective flow meters (PAX-1/8 DIN Digital Input Meter; Red Lion Controls; York, PA) equipped with digital-analog boards (PAXCDL - Analog Output Plug-In Card; Red Lion Controls; York, PA). Data were recorded by PC equipped with a data acquisition board (NI cRIO- 9073, NI 9215, and NI 9237; National Instruments Corporation, Austin, TX) running acquisition software (LabVIEW 8.6.1; National Instruments Corporation; Austin, TX).

Five dedicated pressure transducers (TruWave; Edwards Lifesciences; Irvine, CA) were used to record pressure at the vessel inlets $\left(\mathrm{P}_{\mathrm{A}, \mathrm{i}}\right.$ and $\left.\mathrm{P}_{\mathrm{V}, \mathrm{i}}\right)$, outlets $\left(\mathrm{P}_{\mathrm{A}, \mathrm{o}}\right.$ and $\left.\mathrm{P}_{\mathrm{V}, \mathrm{o}}\right)$, and extravascular space $\left(\mathrm{P}_{\mathrm{EV}}\right)$. These sensors were connected directly to the acquisition board although the PEV signal was amplified by a patient monitor (871 Monitor; Datascope Corporation; MAQUET Incorporated; Bridgewater, NJ). All eight signals were recorded at $10 \mathrm{~Hz}$ throughout the course of each experiment.

Prior to each series of experiments, the chamber and intravascular system were primed and evacuated of air. The arterial inlet and venous outlet pressures $\left(\mathrm{P}_{\mathrm{A}, \mathrm{i}}\right.$ and $\mathrm{P}_{\mathrm{V}, \mathrm{o}}$, respectively) were prescribed (100 and $10 \mathrm{mmHg}$, respectively) by individually adjusting the height of the corresponding reservoirs. The baseline hemodynamics were established with $\mathrm{P}_{\mathrm{EV}}=0 \mathrm{mmHg}$ (in which both vessels were intact and fully patent) and by adjusting $\mathrm{R}_{\mathrm{AV}}$ such that $\mathrm{P}_{\mathrm{V}, \mathrm{i}}=20 \mathrm{mmHg}$. Two types of experiments were performed: 1) a "control" with both vessels fully intact, and 2) a "hemorrhage" where the arterial tube was punctured so as to produce a $1.8 \mathrm{~mm}$ diameter hole approximately midway along its length. For each experiment, the extravascular pressure $\left(\mathrm{P}_{\mathrm{EV}}\right)$ was reduced from 120 to $0 \mathrm{mmHg}$ in $10 \mathrm{mmHg}$ increments. All experiments were repeated five times. For the control experiments, data were collected continuously, and at least 20 seconds were allotted per step to allow stability. For the hemorrhage experiments, $\mathrm{P}_{\mathrm{EV}}$ was initially set with the ball valves closed, isolating the intravascular system. Then, after $\mathrm{P}_{\mathrm{EV}}$ was established, the ball valves were opened and at least 2 seconds of steady-state data were recorded.

At each extravascular pressure level, twenty data points of steady state flows and intravascular pressures were averaged (Table 2). Outliers were removed, defined as $1.5 \mathrm{f}$ from the median where $\mathrm{fs}$ is the difference between lower and upper fourths [24]. Resistances and indices (defined below) were computed from these data.

\section{Lumped-Parameter Model}

An electrical analog model was created to reproduce the experiments. Because the experiments were performed at steadystate, the model consisted exclusively of resistive and voltage elements. Inductive and capacitive elements were not included (Figure 2). The resistive elements were calibrated from the mean measurements of their corresponding pressure drop and flow (Table 3). Due to the collapsibility of the venous tube, its corresponding resistance $\mathrm{R}_{\mathrm{v}}$, was defined as a pressure-dependent resistor via regression of empirical 
Citation: Oberdier MT, Antaki JF (2013) Elevated Pressure Aqueous Hemostasis: Experimental and Mathematical Modeling. J Bioengineer \& Biomedical Sci 3: 126. doi: 10.4172/2155-9538.1000126

Page 3 of 7

data as follows:

$$
R_{V}= \begin{cases}0.1, & P_{T M, V} \leq 0 \\ 7.91 \exp \left(0.060 \cdot P_{T M, V}\right), & 0<P_{T M, V}<P_{C} \\ 9.0 \cdot 10^{-7} \exp \left(0.247 \cdot P_{T M, V}\right), & P_{C} \leq P_{T M, V}\end{cases}
$$

where $\mathrm{P}_{\mathrm{TM}, \mathrm{V}}=\mathrm{P}_{\mathrm{EV}}-\mathrm{P}_{\mathrm{V}}$ is the transmural venous pressure and $\mathrm{P}_{\mathrm{C}}=\mathrm{P}_{\mathrm{A}}-15$ is the critical closing pressure: necessary to fully collapse the venous tube. The three domains of Equation 1 approximately correspond to

\begin{tabular}{|c|c|c|}
\hline Resistance & \multicolumn{2}{|c|}{ Value [mmHg/(L.min)] } \\
\hline$R_{\mathrm{i}}$ & \multicolumn{2}{|c|}{$29 \pm 1.9$} \\
\hline $\mathrm{R}_{\mathrm{A}}$ & \multicolumn{2}{|c|}{$0.94 \pm 0.53$} \\
\hline $\mathrm{R}_{\mathrm{AR}}$ & \multicolumn{2}{|c|}{ See Equation 4.2. } \\
\hline $\mathrm{R}_{\mathrm{VR}}$ & \multicolumn{2}{|c|}{100 (Not Empirical) } \\
\hline $\mathrm{R}_{\mathrm{V}}$ & \multicolumn{2}{|c|}{ See Equation 4.1. } \\
\hline & Control & Hemorrhage \\
\hline $\mathrm{R}_{\mathrm{AV}}$ & $93 \pm 7.1$ & $72 \pm 8.1$ \\
\hline $\mathrm{R}_{\mathrm{o}}$ & $17 \pm 1.8$ & $79 \pm 30$ \\
\hline
\end{tabular}

Table 2: Hydraulic resistance elements of lumped-parameter model. Values determined from experimental data and presented as mean \pm standard deviation.

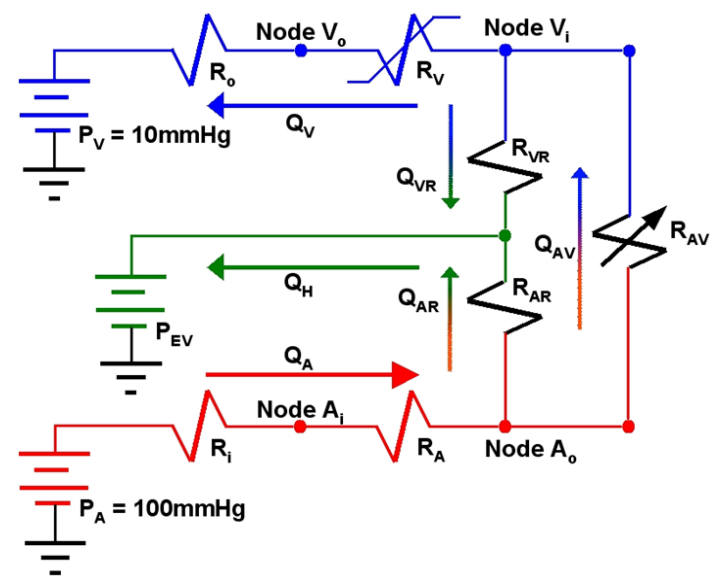

Figure 2: Electrical analog of the experiment complete with pressure sources $\left(P_{A,}, P_{E V}\right.$, and $\left.P_{V O}\right)$, intravascular flows $\left(Q_{A}, Q_{A V}\right.$ and $\left.Q_{V}\right)$, extravascular flows $\left(Q_{A R}, Q_{V R}\right.$, and $\left.Q_{H}\right)$, arterial and venous rents $\left(R_{A R}\right.$ and $R_{V R}$, respectively), arteriovenous resistance $\left(R_{A V}\right)$, and venous resistance $\left(R_{V}\right)$ that varies with transmural pressure. the fully open tube, partial collapse at the venous outlet, and collapse at both the inlet and outlet. The arterial resistance $\mathrm{R}_{\mathrm{AR}}$, also varied with transmural conditions as a result of dilation of the rent, and was regressed via empirical data as:

$$
\mathrm{R}_{\mathrm{AR}}=1.35 . \mathrm{P}_{\mathrm{TM}, \mathrm{A}+} 2.01
$$

where, $\mathrm{P}_{\mathrm{TM}, \mathrm{A}}=\mathrm{P}_{\mathrm{A}}-\mathrm{P}_{\mathrm{EV}}$ is the transmural arterial pressure (Figure 3).

After defining the system parameters, the model was extended to predict results of additional hemodynamic conditions including a venous rent $\left[R_{V R}=100 \mathrm{mmHg} /(\mathrm{L} \cdot \mathrm{min})\right]$ to simulate venous bleeding. Subsequent models included hypotension $\left(\mathrm{P}_{\mathrm{A}}=70 \mathrm{mmHg}\right)$, hypertension $\left(\mathrm{P}_{\mathrm{A}}=130 \mathrm{mmHg}\right)$, limited venous collapse $\left(1 / 2 \mathrm{R}_{\mathrm{V}}\right)$, and simultaneous arterial and venous hemorrhages $\left[\mathrm{R}_{\mathrm{AR}}\right.$ as defined by Equation 2 and $\left.\mathrm{R}_{\mathrm{VR}}=100 \mathrm{mmHg} /(\mathrm{L} \cdot \mathrm{min})\right]$.

The governing equations representing the network in Figure 2 were derived using classical circuit theory, provided in the Appendix. These simultaneous equations were solved using MathCAD software then evaluated using Microsoft Excel.

\section{Characteristic Indices}

The effectiveness of EPAH was evaluated using relationships that quantify: 1) decreased hemorrhage rate, and 2) maintenance of antegrade flow. The Hemorrhage Index is defined as the percent reduction of hemorrhage flow rate due to elevated extravascular pressure:

$$
H I_{P}=\frac{Q_{H, P}}{Q_{H, 0}} \cdot 100
$$

where $\mathrm{Q}_{\mathrm{H}}$ is the hemorrhage flow rate, the subscript 0 refers to the baseline condition $\left(\mathrm{P}_{\mathrm{EV}}=0 \mathrm{mmHg}\right)$ corresponding to maximal hemorrhage $(\mathrm{HI}=100)$, and the subscript $\mathrm{P}$ denotes the elevated extravascular pressure level.

Similarly, the Circulation Index is defined as the percent reduction of continued antegrade flow caused by extravascular pressure:

$$
C I_{P}=\frac{Q_{V, P}}{Q_{V, 0}} \cdot 100
$$

\begin{tabular}{|c|c|c|c|c|c|c|c|c|}
\hline \multirow{2}{*}{$P_{E V, S e t}$} & \multicolumn{4}{|c|}{ Control } & \multicolumn{4}{|c|}{ Hemorrhage } \\
\hline & $\mathrm{P}_{\mathrm{A}, \mathrm{i}}$ & $\mathrm{P}_{\mathrm{A}, \mathrm{O}}$ & $\mathrm{P}_{\mathrm{V}_{\mathrm{i}}}$ & $\mathrm{P}_{\mathrm{v}, 0}$ & $\mathrm{P}_{\mathrm{A}, \mathrm{i}}$ & $\mathrm{P}_{\mathrm{A} .0}$ & $P_{V_{j}}$ & $\mathrm{P}_{\mathrm{V}, 0}$ \\
\hline 0 & $79 \pm 2.9$ & $79 \pm 3.0$ & $19 \pm 1.7$ & $19 \pm 1.7$ & $71 \pm 0.94$ & $71 \pm 1.2$ & $36 \pm 1.3$ & $36 \pm 1.4$ \\
\hline 10 & $81 \pm 1.6$ & $79 \pm 3.1$ & $19 \pm 1.8$ & $19 \pm 1.7$ & $70 \pm 0.04$ & $71 \pm 0.25$ & $30 \pm 8.5$ & $30 \pm 8.3$ \\
\hline 20 & $80 \pm 2.8$ & $80 \pm 2.9$ & $19 \pm 1.7$ & $19 \pm 1.8$ & $73 \pm 1.7$ & $73 \pm 1.15$ & $36 \pm 1.5$ & $36 \pm 1.5$ \\
\hline 30 & $81 \pm 2.9$ & $81 \pm 3.0$ & $25 \pm 0.41$ & $19 \pm 2.0$ & $73 \pm 1.9$ & $74 \pm 1.1$ & $36 \pm 1.3$ & $37 \pm 1.3$ \\
\hline 40 & $84 \pm 2.8$ & $83 \pm 2.9$ & $35 \pm 1.3$ & $17 \pm 1.8$ & $75 \pm 0.74$ & $76 \pm 0.82$ & $37 \pm 0.52$ & $37 \pm 0.36$ \\
\hline 50 & $86 \pm 2.6$ & $86 \pm 2.6$ & $46 \pm 0.12$ & $16 \pm 1.8$ & $78 \pm 0.73$ & $78 \pm 0.48$ & $46 \pm 1.2$ & $36 \pm 0.61$ \\
\hline 60 & $89 \pm 2.3$ & $89 \pm 2.3$ & $56 \pm 0.11$ & $14 \pm 1.7$ & $81 \pm 2.0$ & $82 \pm 1.8$ & $56 \pm 0.52$ & $34 \pm 0.73$ \\
\hline 70 & $91 \pm 2.1$ & $91 \pm 2.1$ & $66 \pm 0.37$ & $13 \pm 1.7$ & $84 \pm 0.96$ & $85 \pm 1.2$ & $65 \pm 1.4$ & $35 \pm 1.4$ \\
\hline 80 & $94 \pm 1.9$ & $93 \pm 2.0$ & $75 \pm 0.10$ & $11 \pm 1.6$ & $88 \pm 0.63$ & $89 \pm 0.43$ & $75 \pm 1.3$ & $32 \pm 0.23$ \\
\hline 90 & $96 \pm 1.6$ & $96 \pm 1.6$ & $84 \pm 0.21$ & $9.8 \pm 1.5$ & $92 \pm 0.87$ & $92 \pm 0.69$ & $83 \pm 0.74$ & $27 \pm 5.6$ \\
\hline 100 & $98 \pm 1.4$ & $98 \pm 1.4$ & $92 \pm 0.38$ & $8.7 \pm 1.3$ & $95 \pm 1.2$ & $96 \pm 0.90$ & $91 \pm 0.63$ & $29 \pm 0.81$ \\
\hline 110 & $99 \pm 1.5$ & $98 \pm 1.5$ & $95 \pm 0.98$ & $8.2 \pm 1.4$ & $100 \pm 1.5$ & $101 \pm 1.2$ & $97 \pm 0.96$ & $25 \pm 5.5$ \\
\hline 120 & $99 \pm 1.6$ & $99 \pm 1.7$ & $96 \pm 1.3$ & $8.0 \pm 1.4$ & $109 \pm 1.4$ & $110 \pm 1.1$ & $106 \pm 0.94$ & $29 \pm 0.57$ \\
\hline
\end{tabular}

where, $Q_{V}$ is the venous flow (distal to an injury). A value of $C I$ equal to 100 corresponds to the baseline hemorrhage condition at $\mathrm{P}_{\mathrm{EV}}=0 \mathrm{mmHg}$.

Table 3: Measured pressures at each node from the control (left) and hemorrhage (right) experiments as a function of extravascular pressure. Data presented as mean \pm standard deviation. 
Citation: Oberdier MT, Antaki JF (2013) Elevated Pressure Aqueous Hemostasis: Experimental and Mathematical Modeling. J Bioengineer \& Biomedical Sci 3: 126. doi: 10.4172/2155-9538.1000126

CI equal to 0 corresponds to cessation of antegrade flow.

The difference between $\mathrm{CI}$ and $\mathrm{HI}$ was introduced to reflect the relative effect of extravascular pressure on circulation versus hemorrhage, termed the Aqueous Hemostasis Index:

$$
A H I_{P}=C I_{P}-H I_{P}
$$

where a value of 0 indicates equal percent reduction in hemorrhage and circulation. A value of 100 would reflect complete cessation of hemorrhage without any reduction of circulation, and -100 would reflect the worst case scenario in which circulation was completely interrupted without any effect on hemorrhage. Resistances are presented as mean \pm standard deviation. Indice values are presented as mean \pm propagated error as determined via arithmetic error propagation functions [25].

\section{Results}

\section{Experiments}

Flow data from both the control and the hemorrhage experiments are presented in Figure 4. For both experiments, the arteriovenous and venous flows were equal $\left(\mathrm{Q}_{\mathrm{AV}}={ }_{\mathrm{QV}}\right)$ within measurement error (data not presented). With regard to control data, the flow rate remained at baseline level until venous collapse was initiated at $20 \mathrm{mmHg}$ (Figure 4a).Above $20 \mathrm{mmHg}$, flows decreased monotonically up to 100 $\mathrm{mmHg}$. For the hemorrhage experiment, antegrade flow (taken as $\mathrm{Q}_{\mathrm{V}}$ ) was nearly constant until extravascular pressure reached $50 \mathrm{mmHg}$. (Figure 4b) Between 50 and $100 \mathrm{mmHg}$, antegrade flow decreased monotonically to approximately $0.0 \mathrm{~L} / \mathrm{min}$. Hemorrhage flow decreased in a similar manner over the full pressure range from 0 to $100 \mathrm{mmHg}$. At approximately $0.2 \mathrm{~L} / \mathrm{min}$ when the extravascular pressure was 90 $\mathrm{mmHg}$, the flow was divided approximately equally between $\mathrm{Q}_{\mathrm{A}}$ and $\mathrm{Q}_{H^{\text {. }}}$ For both experimental conditions, the antegrade flow was completely interrupted when extravascular pressure exceeded arterial pressure.

The Circulation Index increased above baseline from $\mathrm{P}_{\mathrm{EV}}=0$ $\mathrm{mmHg}$ to $10 \mathrm{mmHg}$ where the extravascular pressure was insufficient to initiate venous collapse (Figure 5). Throughout the remainder of the extravascular pressure range, hemorrhage was suppressed while antegrade circulation was diminished but sustained. The Aqueous Hemostasis Index increased above baseline corresponding to suppressed hemorrhage with sustained circulation less than $\mathrm{P}_{\mathrm{EV}}=90 \mathrm{mmHg}$. At this level and above, the Aqueous Hemostasis Index values were below zero.

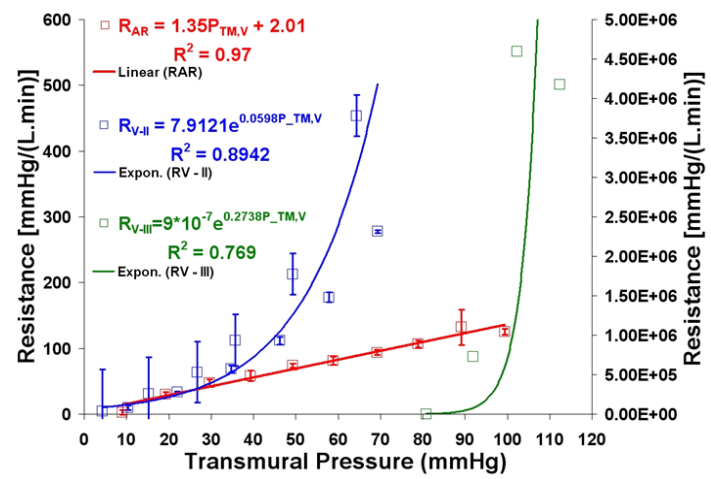

Figure 3: Experimental data and corresponding regressions for venous resistance $\left(R_{v}\right)$ and arterial rent resistance $\left(R_{A R}\right)$. Transmural pressures were taken as $\left(P_{E V Y V}\right)$ and $\left(P_{A P E V}\right)$ for $R_{V}$ and $R_{A R}$, respectively. The $R V-I I$ and $R_{V}-I I I$ data refer to the left and right axes, respectively, and are components of the piecewise function of Equation 1. Standard deviations for $\mathrm{R}_{\mathrm{v}}-\mathrm{III}$ were too large to be displayed and from left to right are $90.0,1.45 \times 10^{\wedge} 6.06 \times 10^{\wedge} 6$, and $1.13 \times 10^{\wedge} 7$, respectively.
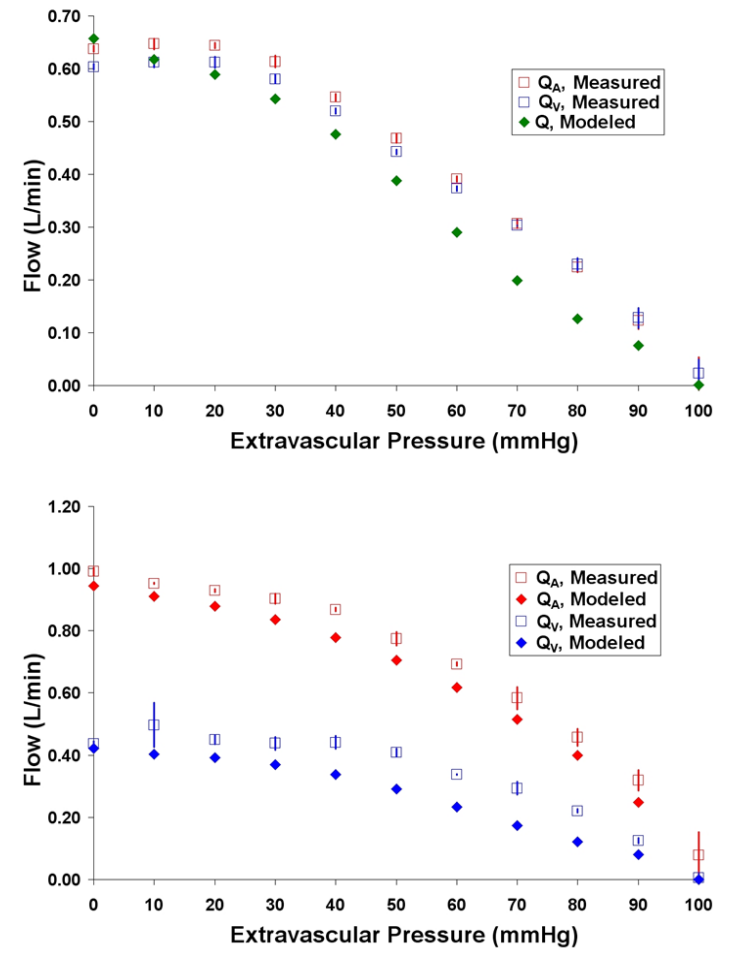

Figure 4: Validation of lumped-parameter model. Flow rates from the control (top) and hemorrhage (bottom) experiments. Experimental (solid) flows are in general agreement with modeled (checkered) flows. Extravascular pressure $\left(P_{E V}\right)$ is the independent parameter. Data presented as mean \pm standard deviation.

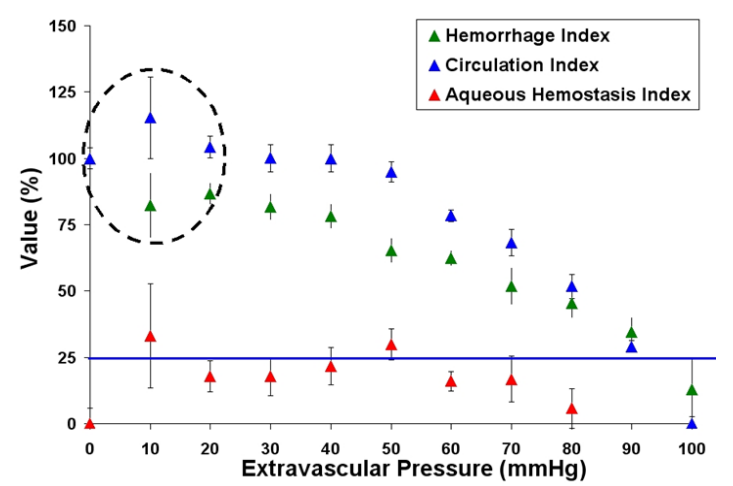

Figure 5: EPAH indices from the experiment with extravascular pressure $\left(P_{E Y}\right)$ as the independent variable. The blue horizontal line is a threshold of maintained antegrade flow. Note the increase in antegrade flow (circle) at $P_{E V}=10 \mathrm{mmHg}$. Data presented as mean \pm propagated error.

\section{Lumped-Parameter Modeling}

The hemodynamic conditions from the experiment were reproduced with the lumped-parameter model (Figures 4 and 6). Experimental and modeled flows closely correspond as indicated by mean squared error values of $1.31 \times 10^{-1}$ and $1.28 \times 10^{-1}$ for the control and hemorrhage models, respectively. For the control conditions, the model underestimated flow when $\mathrm{P}_{\mathrm{EV}} \geq 20 \mathrm{mmHg}$ whereas with hemorrhage, the model underestimated flows throughout the range of extravascular pressure. 

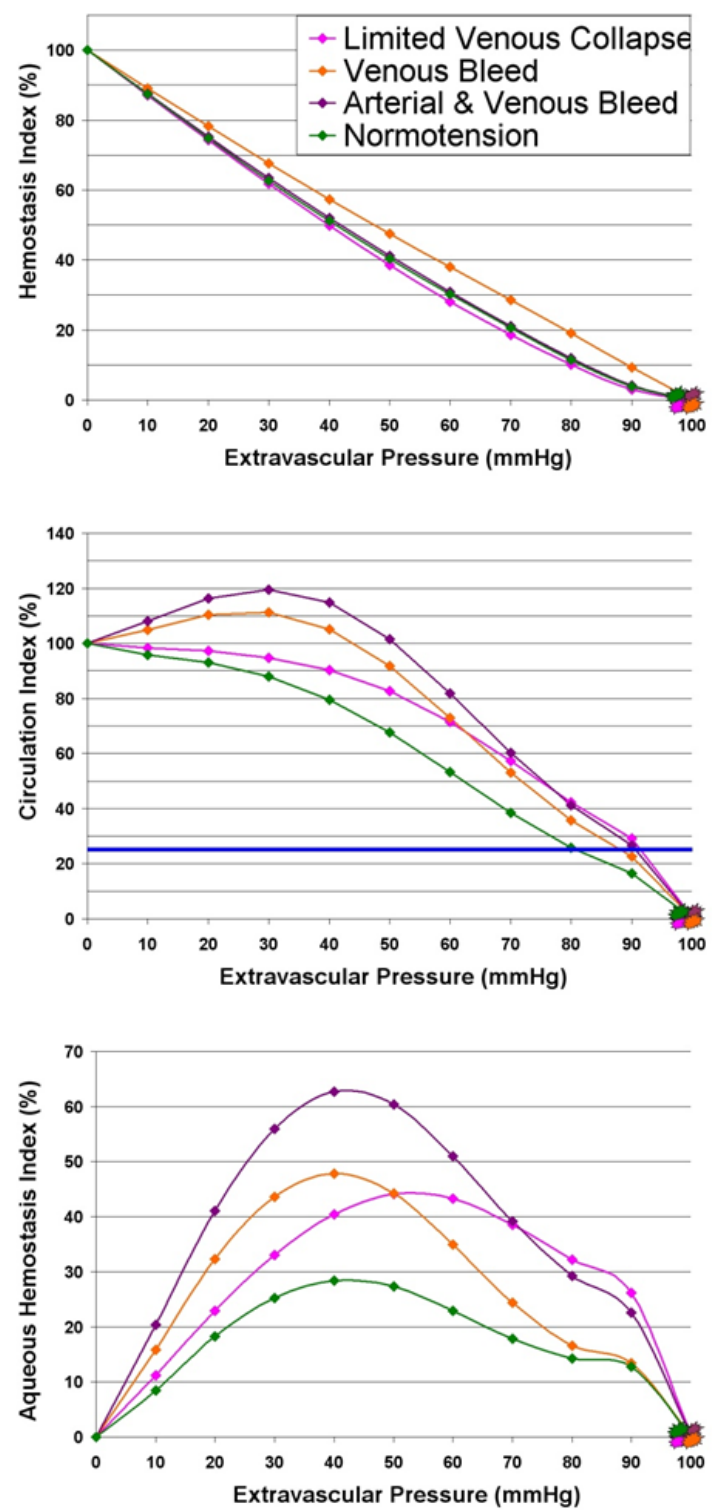

Figure 6: Indices of hemostasis and circulation as a function of extravascular pressure determined by lumped-parameter models. The "Normotension" model was derived utilizing the hemodynamic settings from the experiment. The blue horizontal lines are thresholds of maintained antegrade flow. Stars indicate the extravascular pressure level at which the corresponding indices reduce to zero.

For the limited venous collapse model, Circulation Indices were greater at all extravascular pressure steps compared to the normotension model. Further, for the venous bleed and simultaneous arterial and venous bleed models, the Circulation Indices were predicted to increase above baseline up to PEV $<50 \mathrm{mmHg}$. For these models, Aqueous Hemostasis Index values remained above zero until the extravascular pressure step corresponding to complete venous collapse. For the hypo- and hypertension models, Hemostasis Indices were comparable to results for the other models while the Aqueous Hemostasis Indices reduced to zero above $80 \mathrm{mmHg}$ and less than $70 \mathrm{mmHg}$, respectively. Circulation Indices for the hypotension case did not follow a consistent trend with increasing extravascular pressure. (Hypo- and hypertension data not shown)

\section{Discussion}

The EPAH technique is anticipated to be useful for particularly bloody procedures such as those for trauma, aneurysm repair, arteriovenous malformation removal, or tumor resection. Use of this technique would provide the surgeon with more time to devote to the technical aspects of the procedure by reducing or eliminating the need for vascular clipping, cautery, and suturing to control bleeding. Also, a more attentive surgeon and shorter operating times would likely improve patient outcomes.

Although the suppression of hemorrhage with elevated extravascular aqueous pressure has been reported in the clinical literature $[4,5,9,10]$, the studies here are the first to quantify this capability. Iglesias et al. $[4,5]$ observed that during transurethral resection of the prostate, intravesicoprostatic pressures higher than $10 \mathrm{mmHg}$ distorted the normal anatomy of vascular structures, which is consistent with the finding here that elevated extravascular pressure above venous pressure causes venous collapse. During the procedure, "hydraulic hemostasis" was achieved with a pressure of $90 \mathrm{~cm} \mathrm{H}_{2} \mathrm{O}(66 \mathrm{mmHg})$ although the technique was complicated by the absorption of electrolyte-free irrigant via the open, cut vessels. In the venous bleed and simultaneous arterial and venous bleed models presented here, intravasation of fluid was predicted and contributed to antegrade flow as indicated by Circulation Indices greater than baseline for $10 \leq \mathrm{PEV} \leq 40 \mathrm{mmHg}$. Jensen et al. [10] also demonstrated clinically that a pressure of $55 \mathrm{mmHg}$ could be applied during shoulder surgery without detriment while facilitating visualization and the control of bleeding. Also during arthroscopic shoulder surgery, Morrison et al. [9] showed that pressurization of the subacromial space below systolic blood pressure by $49 \mathrm{mmHg}$ was sufficient to arrest arteriolar and capillary bleeding. This was deemed significant because of the theoretical relationship to blood pressure and critical closing pressure.

The experimental system is a collapsible tube model that has been well- characterized [26,27]. Further, the experiment involved collapsible tubes connected in series, which is a configuration that has also been studied in great detail $[28,29]$. However, the control experiments were unique in their use of simultaneously prescribed arterial and venous hemodynamics. Consistent with reports from the literature (e.g. [30]), self-excited oscillations (flutter) in the venous tube were occasionally observed during the control experiment when the extravascular pressure was in the domains of 30 and $40 \mathrm{mmHg}$. Flutter was not observed during the hemorrhage experiments.

The experiments also demonstrated that the venous critical closing pressure determines cessation of antegrade flow, and there is not a benefit to increasing extravascular pressure beyond arterial pressure. Therefore, arterial collapse is an unimportant consideration for EPAH. Nevertheless, data showed that an arterial hemorrhage could not be completely suppressed while antegrade flow was sustained. Therefore, the use of EPAH to arrest arterial bleeding will likely cause venous collapse in the immediate vicinity. However, this does not negate the possibility of intermittent EPAH at arterial pressure for short periods of time. As extreme examples, highly metabolic organs such as the brain and heart are able to tolerate circulatory shock for up to 5 and 20 minutes, respectively, without permanent damage [31]. This would likely be enough time for the surgeon to emergently arrest bleeding with EPAH, clear the visual field, and repair the injury before lowering the pressure to allow reperfusion. Hence, the clinical implementation of EPAH could involve an intermittent extravascular pressure profile that is determined by the metabolic demands of the surrounding tissues. 
With particular regard to cranial EPAH, a sustained antegrade flow reduction to approximately $25 \%$ of normal was shown to be necessary to cause hypoxia [32]. However, thresholds of cerebral ischemia (e.g. $[33,34]$ ) and normal cerebral blood flows (e.g. [35]) vary depending on the method of measurement.

A fortuitous observation from the experiments is the remarkable effect of merely $10 \mathrm{mmHg}$ extravascular pressure for both diminishing hemorrhage and increasing antegrade flow. Further observations include that increasing extravascular pressure to $80 \mathrm{mmHg}$ corresponded to a Hemorrhage Index of $46 \%$ and a Circulation Index of 52\%. Also, the Aqueous Hemostasis Index became less than zero and the Circulation Index dropped below the threshold of $25 \%$ at $90 \mathrm{mmHg}$. Therefore, it would be reasonable to define an optimal (safe and effective) domain of continuous extravascular pressure from 10 to less than $90 \mathrm{mmHg}$, albeit for this particular set of conditions. Extending this observation to other situations may be performed ad-hoc, in which the indices of hemostasis and perfusion are used to define upper and lower bounds of EPAH pressure.

As demonstrated via the experiments, the Aqueous Hemostasis Index simultaneously captured changes in hemorrhage and antegrade flow. For the normotension, limited venous collapse, venous hemorrhage, and simultaneous arterial and venous hemorrhage models, a transition in this index from positive to negative values closely corresponded to safe and effective pressure domains identified using the aforementioned rationale. Hence, the Aqueous Hemostasis Index may serve as a concise means of determining boundaries of EPAH pressure. Nonetheless, difficulties in accurately modeling hypo- and hypertension suggest that additional experiments would be necessary to accurately calibrate these altered loading conditions. Ideally, a tube law would be utilized to model the resistance of venous collapse.It may seem counterintuitive to rely on an empirical relationship for the current lumped-parameter models in light of the numerous mathematical models that already exist for tube collapse. However, this was found to be necessary because inlet and outlet pressure were both dependent variables in these studies whereas tube laws presume a known transmural pressure (e.g. [36,37]).

Several simplifications were made for this study including time invariance, unsupported vessels, and an idealized rent. Although pulsatility is a significant feature of blood flow in large vessels, time invariance was chosen to quantify EPAH without the confounding effects of compliance and inertance. However, dynamic similarity was maintained such that the Reynolds numbers of the arterial and venous conduits were comparable to those of the abdominal aorta and inferior vena cava (for the control experiment with $\mathrm{P}_{\mathrm{EV}}=0 \mathrm{mmHg}$ ) [20]. Additionally, the vessels of the experiments were suspended in an aqueous medium without tethering along their length. In most circumstances, unless the vessel has been completely dissected, surrounding tissue will provide additional stress shielding against collapse. That is, deep vessels may not be affected by elevated superficial pressure. In addition to collateral circulation, this stress shielding may ameliorate the adverse effects of localized hemostatic pressure. Results of the limited venous collapse model suggest that a tethered vessel may permit more antegrade flow for a given extravascular pressure. Further, the punched circular hole does not necessarily represent a generic vascular wound. Instead, the independent variable of interest for this study was its hemodynamic resistance, irrespective of geometry.

Further investigations may include improved physiologically-based lumped-parameter models that feature pulsatility and autoregulation (e.g. [38]). Finite element models would be insightful for studying the effect of EPAH on compression of underlying vasculature embedded in tissue. Ultimately, in-vivo models would be required to definitively assess histopathologic damage from sustained elevated extravascular aqueous pressure. Such studies would incorporate physiologic characteristics that were not replicated by the experiments including pulsatility, a capillary network, collateral flow, autoregulation, and metabolism - all relevant to the safety and efficacy of EPAH.

\section{Conclusions}

Experiments were performed with arterial and venous pressure set at 100 and $10 \mathrm{mmHg}$, respectively. Relative to these prescribed conditions, experiments showed that hemorrhage could be diminished with increasing steps of extravascular pressure although above $40 \mathrm{mmHg}$, antegrade flow decreased due to venous collapse. At an extravascular pressure of $10 \mathrm{mmHg}$, the hemorrhage was decreased while antegrade flow was fully maintained. Above $80 \mathrm{mmHg}$, antegrade flow dropped below a threshold of $25 \%$ of normal. Above arterial pressure, flow ceased. Therefore, for the hemodynamic conditions prescribed in the experiments, it would be reasonable to define an optimal (safe and effective) domain of continuous extravascular to be from 10 to less than $90 \mathrm{mmHg}$.

Utilizing an electric analog of the experimental circuit, lumpedparameter models of normotension, limited venous collapse, venous hemorrhage, and simultaneous arterial and venous hemorrhages were established. For these models, a transition in the Aqueous Hemostasis Index from positive to negative values closely corresponded to safe and effective pressure domains identified using the aforementioned rationale. Hence, the Aqueous Hemostasis Index may serve as a more concise means of determining boundaries of EPAH pressure. Further, the limited venous collapse model indicates that a tethered vein may permit more antegrade flow for a given extravascular pressure. Also, the venous bleed and simultaneous arterial and venous bleed models suggest that elevated extravascular pressure may cause venous intravasation. Finally, additional experiments would be necessary to accurately calibrate the altered loading conditions of hypo- and hypertension.

\section{Lumped-Parameter Model}

The governing relationships involved in the resistive model are analogs of Ohm's and Kirchoff's laws: $\Delta \mathrm{P}=\mathrm{QR}$ and $\Sigma \mathrm{Q}=0$, respectively. When combined, those mathematical definitions specific to Nodes $A_{i}$, $A_{o}, V_{i}$, and $V_{o}$ of the experiment respectively yield:

$$
\begin{aligned}
& \frac{P_{A}-P_{A, i}}{R_{i}}-\frac{P_{A, i}-P_{A, o}}{R_{A}}=0 \\
& \frac{P_{A, i}-P_{A, o}}{R_{A}}-\frac{P_{A, o}-P_{E V}}{R_{A R}}-\frac{P_{A, o}-P_{V, i}}{R_{A V}}=0 \\
& \frac{P_{A, o}-P_{V, i}}{R_{A V}}-\frac{P_{V, i}-P_{E V}}{R_{V R}}-\frac{P_{V, i}-P_{V, o}}{R_{V}}=0
\end{aligned}
$$$$
\text { and } \frac{P_{V, i}-P_{V, o}}{R_{V}}-\frac{P_{V, o}-P_{V}}{R_{o}}=0
$$

where pressures and resistances were previously defined, and $\mathrm{P}_{\mathrm{A}, \mathrm{i},}$ $\mathrm{P}_{\mathrm{A}, \mathrm{o}}, \mathrm{P}_{\mathrm{V}, \mathrm{o}}$, and $\mathrm{P}_{\mathrm{V}, \mathrm{i}}$ were the unknowns in the system. 
Citation: Oberdier MT, Antaki JF (2013) Elevated Pressure Aqueous Hemostasis: Experimental and Mathematical Modeling. J Bioengineer \& Biomedical Sci 3: 126. doi: 10.4172/2155-9538.1000126

\section{Acknowledgements}

The authors thank Abhinav Singhal for coding the data acquisition software and Antonio Ferreira for assistance with solving the lumpedparameter model. This work was supported by a grant from the National Institute of General Medical Sciences (F31GM089135) to MTO.

\section{References}

1. Melman L, Matthews BD (2008) Current trends in laparoscopic solid organ surgery: spleen, adrenal, pancreas, and liver. Surg Clin North Am 88: 10331046, vii.

2. Wright DM, et al. (1996) Extraperitoneal carbon dioxide insufflation. Anesthesia and Analgesia 83: 193-194.

3. Iglesias JJ, Fiore G (1975) Iglesias resectoscope with simultaneous irrigation, suction and low intravesical pressure. Eur Urol 1: 251-254.

4. Iglesias JJ, Perez-Castro EE, Massuri SD, Sporer A, Seebode JJ (1977) Hydraulic hemostasis in transurethral resection of the prostate using the Iglesias continuous suction resectoscope. J Urol 117: 306-308.

5. Iglesias JJ, Ellendt EP, Pettirossi O, Madduri SD, Sporer A, et al. (1977) The importance of continuous pump suction in TUR hydraulic hemostasis. Eur Uro 3: 150-153.

6. Baumann R, Magos AL, Kay JD, Turnbull AC (1990) Absorption of glycine irrigating solution during transcervical resection of endometrium. BMJ 300: 304-305

7. Maier RC (1993) Control of postpartum hemorrhage with uterine packing American Journal of Obstetrics and Gynecology 169: 317-323.

8. Vilos GA, Edris F (2007) Second-generation endometrial ablation technologies: the hot liquid balloons. Best Pract Res Clin Obstet Gynaecol 21: 947-967.

9. Morrison DS, Schaefer RK, Friedman RL (1995) The relationship between subacromial space pressure, blood pressure, and visual clarity during arthroscopic subacromial decompression. Arthroscopy 11: 557-560.

10. Jensen $\mathrm{KH}$, et al. (2001) Arthroscopic shoulder surgery with epinephrine saline irrigation. Arthroscopy 17: 578-581.

11. Nakano T, Ohkuma H, Ebina K, Suzuki S (2003) Neuroendoscopic surgery for intracerebral haemorrhage--comparison with traditional therapies. Minim Invasive Neurosurg 46: 278-283.

12. Anderson RCE, Walker ML (2008) Neuroendoscopy, in Principles and Practice of Pediatric Neurosurgery, Albright AL, Adelson PD, Pollack IF (Editors) Thime Medical Publisher: New York, USA.

13. Dandy WE (1922) Cerebral Ventriculoscopy. Bulletin of the Johns Hopkins Hospital 33: 189

14. Mixter WJ (1923) Ventriculoscopy and puncture of the floor of the third ventricle. Boston Medical and Surgical Journal 188: 277-278.

15. Nagasaka T, Tsugeno M, Ikeda H, Okamoto T, Takagawa $\mathrm{Y}$, et al. (2009) Balanced irrigation-suction technique with a multifunctional suction cannula and its application for intraoperative hemorrhage in endoscopic evacuation of intracerebral hematomas: technical note. Neurosurgery 65: E826-827.

16. Bakshi A, Bakshi A, Banerii AK (2004) Neuroendoscope-assisted evacuation of large intracerebral hematomas: introduction of a new, minimally invasive technique. Preliminary report. Neurosurg Focus 16: e9.

17. Chen CC, Cho DY, Chang CS, Chen JT, Lee WY, et al. (2005) A stainless stee sheath for endoscopic surgery and its application in surgical evacuation of putaminal haemorrhage. J Clin Neurosci 12: 937-940.

18. Hsieh PC, Cho DY, Lee WY, Chen JT (2005) Endoscopic evacuation of putaminal hemorrhage: how to improve the efficiency of hematoma evacuation. Surg Neurol 64: 147-153.

19. Fung YC (1997) Biomechanics: Circulation, Springer-Verlag, New York, USA.

20. Brodkey RS, Hershey HC (1988) Transport Phenomena: A Unified Approach. McGraw-Hill, Boston, USA.

21. Conrad WA (1969) Pressure flow relationship in collapsible tubes. IEEE Transactions on Biomedical Engineering 16: 284-295.

22. Raju S, et al. (1998) Tube collapse and valve closure in ambulatory venous pressure regulation: Studies with a mechanical model. Journal of Endovascular
Surgery 5: 42-51.

23. Katz Al, Chen Y, Moreno AH (1969) Flow through a collapsible tube. Experimental analysis and mathematical model. Biophysical Journal 9: 1261 1279.

24. Devore JL (2000) Probability and Statistics for Engineering and the Sciences. 5 th ed. Duxbury, Australia.

25. Ku HH (1966) Notes on the use of propagation of error formulas. Journal of Research of the National Bureau of Standards - C. Engineering and Instrumentation 70: 263-273.

26. Kamm RD, Pedley TJ (1989) Flow in collapsible tubes: a brief review. J Biomech Eng 111: 177-179.

27. Shapiro AH (1997) Steady flow in collapsible tubes. Journal of Biomechanical Engineering 99: 126-147.

28. Elad D, et al. (1992) Steady flow through collapsible tubes: Measurements of flow and geometry. Journal of Biomechanical Engineering 114: 84-91.

29. Griffiths DJ (1975) Negative-resistance effects in flow through collapsible tubes: 1 Relaxation oscillations. Medical \& Biological Engineering 13: 785-790.

30. Jensen OE (1992) Chaotic oscillations in a simple collapsible-tube model Journal of Biomechanical Engineering 114: 55-59.

31. Holcroft JW, Blaisdell FW (1991) Shock: Causes and Management of Circulatory Collapse, in Textbook of Surgery: The Biological Basis of Modern Surgical Practice, Sabiston DC (Ed) W.B. Saunders Company: Philadelphia, USA.

32. Morawetz RB, Crowell RH, DeGirolami U, Marcoux FW, Jones TH, et al. (1979) Regional cerebral blood flow thresholds during cerebral ischemia. Fed Proc 38: 2493-2494.

33. Astrup J, Siesjö BK, Symon L (1981) Thresholds in cerebral ischemia - the ischemic penumbra. Stroke 12: 723-725.

34. Arakawa S, Wright PM, Koga M, Phan TG, Reutens DC, et al. (2006) Ischemic thresholds for gray and white matter: a diffusion and perfusion magnetic resonance study. Stroke 37: 1211-1216.

35. Wilkinson IM, et al. (1969) Regional blood flow in the normal cerebra hemisphere. Journal of Neurology, Neurosurgery, and Psychiatry 32: 367-378.

36. Oates GC (1975) Fluid flow in soft-walled tubes. Part I: Steady flow. Medical \& Biological Engineering 13: 773-779.

37. Brower RW, Noordergraaf A (1973) Pressure-flow characteristics of collapsible tubes: A reconciliation of seemingly contradictory results. Annals of Biomedical Engineering 1: 333-355.

38. Ursino $\mathrm{M}$, Lodi $\mathrm{CA}, \mathrm{A}$ simple mathematical model of the interaction between intracranial pressure and cerebral hemodynamics. Journal of Applied Physiology 82: 1256-1269. 